\title{
The colorectal carcinoma prognosis factors. Significance of diagnosis delay
}

\author{
E. Gómez-Domínguez, M. Trapero-Marugán, A. J. del Pozo, J. Cantero, J. P. Gisbert and J. Maté \\ Service of Gastroenterology. Hospital Universitario de La Princesa. Madrid, Spain
}

\begin{abstract}
Introduction: detection of early-stage colorectal carcinoma (CRC) -( Dukes' A or B)- provides better survival rates in these patients. Thus, the effectiveness of screening programs in asymptomatic patients or of early diagnosis in symptomatic individuals has been postulated. The aim of this study was to establish whether a delay in diagnosis or other factors are related to CRC stage.

Patients and methods: a retrospective study was performed on 96 patients with CRC. Age at diagnosis, gender distribution, intestinal disorders, diagnosis delay, primary sign and -regarding CRC- localization, stage (Dukes') and grade of differentiation (well differentiated; non-well differentiated; poorly differentiated) were recorded.

Results: diagnosis delay was $185 \pm 190$ days. Patients delay in obtaining a diagnosis was $119 \pm 158$ days. In $40 \%$ of patients CRC was diagnosed at an early stage (Dukes' A or B), and in $13 \%$ $\mathrm{CRC}$ was poorly differentiated. The only factor with an independent effect on Dukes' stage was tumor differentiation (p: 0.0012). Distal location was associated with less advanced tumors without statistical significance (p: 0.156).

Conclusion: based on the presented data, a greater effort regarding screening programs for healthy people seems warranted, as improved survival has been demonstrated when diagnosis delay is reduced, particularly in patients with the highest mean delay.
\end{abstract}

Key words: Colorectal cancer. Diagnostic delay. Degree of tumor differentiation.

Gómez-Domínguez E, Trapero-Marugán M, Del Pozo AJ, Cantero J, Gisbert JP, Maté J. The colorectal carcinoma prognosis factors. Significance of diagnosis delay. Rev Esp Enferm Dig 2006; 98: 322-329.

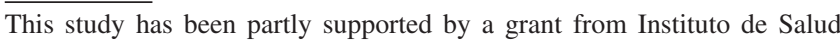
Carlos III (C03/02).

Recibido: $17-12-04$.

Aceptado: 29-12-05.

Correspondencia: Elena Gómez Domínguez. Hospital Universitario de La Princesa. C/ Diego de León, 62. 28006 Madrid. Fax: 914022 99. e-mail: elenagodo@hotmail.com

\section{INTRODUCTION}

Detection of early-stage colorectal carcinoma (CRC) -(Dukes' A or B)- provides $85 \%$ 5-year survival rates in these patients (1). Two ways have been proposed to reach an early diagnosis: tumor diagnosis during the asymptomatic period and reduction of diagnostic delay. Public health policies in Western countries such as the United Kingdom have established screening programs in which symptomatic patients may be assisted by a gastroenterologist within two weeks (2). This is a cheaper option, but its efficacy is unknown (3-5). Furthermore, there is no consensus as to which other factors may be associated with tumor extension (6-10).

The primary aim of this study was to determine the influence of diagnostic delay on tumor extension, and whether patients with CRC may benefit from shorter diagnostic delays. Secondly, our aim was to establish whether any other factors have an influence on disease stage at the time of diagnosis.

\section{MTERIAL AND METHODS}

-Design. A prospective study following patients diagnosed with colorectal cancer in the Endoscopy Unit of a $3^{\text {rd }}$-level hospital. Demographic issues were compiled by personal interviews during endoscopic procedures.

-Patients. One hundred and ten subjects were enrolled; they had been previously diagnosed with colorectal cancer by means of colonoscopy and biopsy. A surgical specimen was obtained in all cases.

-Size. We collected data for 110 consecutive patients, aiming at a sample size of 100 individuals. It was previously considered that, in up to $10 \%$ of patients, the surgical specimen would not be reliable to analyze, due to unresectability or excision in another hospital. 
-Collected variables. Besides cancer location, obtained by colonoscopy, other features were compiled:

1. By face-to-face interview during diagnosis: age, sex, bowel habit, laxative usage, and diagnosis delay.

2. By studying the resected specimen: tumor extension and tumor differentiation grades.

-Definitions. Primary sign or symptom related to the disease: hematochezia, fecal occult blood, ferropenic anemia in the absence of other hemorrhagic lesions, palpable abdominal mass, or change in bowel habit.

- Diagnosis delay: period of time elapsed from first colorectal symptom to endoscopic diagnosis, divided into three components:

1. Patient-related delay: period of time from initial symptom to presentation.

2. Physician-related delay: time wasted by physician until colonoscopy is recommended.

3. Administration-related delay: time elapsed from colonoscopy indication to colonoscopy performance.

- Tumor location: the colon was anatomically divided into: rectal ampulla (from the pectineous line to the beginning of the sigmoid colon); left colon (from the rectal ampulla to the splenic flexure); transverse colon (from the splenic flexure to the hepatic flexure); and right colon (ascending colon and cecum).

- Tumor extension (Dukes' scale): a) tumor growth that disrupts the basal membrane but does not involve the submucosa; b) tumor extension that involves all intestinal layers except the serosal one; c) tumor growth that extends beyond the intestinal wall and involves lymph nodes; and d) distant metastases.

- Tumor differentiation expressed as: well-differentiated, moderately-differentiated and poorly-differentiated tumor, according to number of glands and the presence of morphologic aberrations. Tumors with mucinous material or signet-ring cells in more than $70 \%$ of its volume were labeled as coloid tumors.

-Statistical study. A Chi-squared test was used to compare percentages. A significance level of $p<0.05$ was previously established. A multivariate analysis of data obtained was also performed.

\section{RESULTS}

Data for 109 patients were compiled, but a surgical specimen could only be studied in 96, and tumor extension in 99 . Twelve patients were lost -8 because of unresectability 4 because excision had been performed in another center. One patient was excluded after being diagnosed with colorectal melanoma.

Fifty-four percent of our patients were male, with a mean age of $64 \pm 18$ years. The primary symptom on presentation was hematochezia (40\%), followed by change in bowel habit (23\%) and tenesmus (20\%). Forty percent of patients were Dukes' A or B, and 13\% were poorlydifferentiated. Total delay in diagnosis was $185 \pm 190$ days, with $119 \pm 158$ days being related to patient delays in seeking help. The physician-related delay was $38 \pm 78$ days, and the one related to clerical causes $28 \pm 27$ days. More than half of subjects had their diagnosis delayed between $155 \pm 151$ and $265 \pm 338$ days, with more than a half being attributed to the patient $(177 \pm 245$ days $)$. Table I summarizes patient demographic and description data for those who completed the study. Histological differentiation was the only variable that showed statistical significance in association with tumor extension $(\mathrm{p}<0.05)$. Distal location was associated with lower Dukes' stages, but with no statistical significance $(\mathrm{p}=0.01)$. A significant association between tumor location and histologic type could not be demonstrated. The attached tables II-IV show the statistical association of studied variables with tumor extension.

Table I. Epidemiological and demographic variables

\begin{tabular}{lc}
\hline Age (mean \pm SD) & $64 \pm 18$ \\
\hline Sex (\% male) & 54 \\
\hline Constipation (\%) & 29 \\
\hline Laxatives (\%) & 15 \\
\hline First symptom (\%) & 40 \\
Rectorrhage & 21 \\
Anemia & 23 \\
Change in bowel habit & 20 \\
Tenesmus & \\
\hline Tumor location (\%) & 16 \\
Rectum & 41 \\
Left colon & 34 \\
Transverse colon & 4 \\
Right colon & \\
\hline Delay (days) (mean \pm SD) & $185 \pm 191$ \\
Global & $119 \pm 158$ \\
Patient's delay & $38 \pm 78$ \\
Physician's delay & $28 \pm 27$ \\
Administration's delay & \\
\hline Differentiation (\%) & 15 \\
Well-differentiated & 68 \\
Moderately-differentiated & 13 \\
Poorly-differentiated & 39 \\
\hline Dukes'stages (\%) & 32 \\
$\quad$ A & 22 \\
$\quad$ B & \\
$\quad$ D & \\
\hline SD: standard devition & \\
\hline
\end{tabular}

SD: standard deviation.

\section{DISCUSSION}

Grade of tumor differentiation is the only prognostic value significantly influencing tumor extension in this study. Other patient-related variables (age, gender...) or illness characteristics (i.e., presentation symptom) do not 
Table II. Statistical association between tumor extension and first symptom $(p=\mathbf{0 . 6 6 8})$

\begin{tabular}{|c|c|c|c|c|c|c|}
\hline Tumor extension & Changed habit & Anemia & Tenesmus & Rectorrhage & Other & Total \\
\hline Dukes A & & $1(5.3 \%)$ & $1(5 \%)$ & $5(12.5 \%)$ & & $7(7 \%)$ \\
\hline Dukes B & $4(20 \%)$ & $8(42 \%)$ & $9(45 \%)$ & $17(42.5 \%)$ & $1(25 \%)$ & $39(38 \%)$ \\
\hline Dukes C & $9(45 \%)$ & $7(37 \%)$ & $5(25 \%)$ & $10(25 \%)$ & $2(50 \%)$ & $32(33 \%)$ \\
\hline Total & $20(100 \%)$ & $19(100 \%)$ & $20(100 \%)$ & $40(100 \%)$ & $4(100 \%)$ & $100(100 \%)$ \\
\hline
\end{tabular}

Table III. Statistical association between tumor extension and location $(p=\mathbf{0 . 1 5})$

\begin{tabular}{lccccc}
\hline Tumor extension & Rectum & Left colon & Transverse colon & Right colon & Total \\
\hline Dukes A & 0 & $3(7.3 \%)$ & $1(3 \%)$ & 1 & $3(25 \%)$ \\
Dukes B & $5(31.3 \%)$ & $11(27 \%)$ & $19(56 \%)$ & $1(25 \%)$ & $36(40 \%)$ \\
Dukes C & $9(56.3 \%)$ & $15(36.5 \%)$ & $8(23.5 \%)$ & $2(50 \%)$ & $22(23 \%)$ \\
Dukes D & $2(12.5 \%)$ & $12(29.3 \%)$ & $6(8 \%)$ & $4(100 \%)$ & $95(100 \%)$
\end{tabular}

Table IV. Statistical association between tumor extension, diagnostic delay, histologic differentiation, age, and sex

\begin{tabular}{|c|c|c|c|c|c|c|}
\hline & \multicolumn{5}{|c|}{ Dukes' stages } \\
\hline & & $A$ & $B$ & C & $D$ & $p$ \\
\hline Age & & $64 \pm 18$ & $66 \pm 11$ & $68 \pm 11$ & $66 \pm 12$ & n.s. \\
\hline Global delay (days) & & $78 \pm 66$ & $155 \pm 151$ & $265 \pm 338$ & $135 \pm 152$ & n.s. \\
\hline Patient's delay (days) & & $74 \pm 61$ & $83 \pm 98$ & $177 \pm 245$ & $101 \pm 146$ & n.s. \\
\hline Administration's delay (days) & & $15 \pm 15$ & $28 \pm 26$ & $36 \pm 55$ & $20 \pm 20$ & n.s. \\
\hline Histologic & Well- Dif. & $3(75 \%)$ & $4(11 \%)$ & $4(12 \%)$ & $4(17 \%)$ & \\
\hline differentation & Mod-.Dif. & & $30(83 \%)$ & $23(70 \%)$ & $15(65 \%)$ & 0.012 \\
\hline Patients (\%) & Poor- Dif. & $1(25 \%)$ & $2(6 \%)$ & $6(18 \%)$ & $4(17 \%)$ & \\
\hline
\end{tabular}

n.s.: no significance.

seem to modify tumor extension. There is only a slight association between tumors in a distal localization and early tumor stages, but with no statistically significant value, probably due to a type $\beta$ error. Otherwise, delay in $\mathrm{CRC}$ diagnosis has not been related to greater disease extension.

All these data do not mean that, individually, patients had not benefited from earlier diagnosis. Probably, some variable, as illness severity or immunotolerance, is confounding the importance of diagnosis delay when a group rather than individual patients is studied.

It is important to remark that more than half of diagnoses occur after 5 months, and almost $30 \%$ at 9 months. These rates are worse than other published data (3); nevertheless, they are better than those registered by our group during 1978-82 (5). Nearly $70 \%$ of diagnosis delay is imputable to the patient, in a similar way as other pub- lished studies have pointed out $(2,3)$. The main reason for not visiting a doctor could be a lack of symptom recognition. Some other times, symptoms are very mild (i.e., occult blood in feces), and these are only recognized during a routine medical examination.

As other authors suggest, diagnosis delay is not a significant variable related to prognosis and tumor extension $(2-5,11)$. Nevertheless, a positive relationship between histologic type and grade, and tumor extension has been found $(5,6,11,12)$. The main problem is the poor objectivity of tumor classification using differentiation grades, so that more than $50 \%$ of tumors are classified as moderately differentiated. In our series, tumors with lower differentiation grades represented $13 \%$, similar to other studies (13) and in contrast to Japanese series, where these less differentiated tumors appear in at least $25 \%$ of cases (11). 
Some authors hold that patients with better differentiation and older age have a more favorable prognosis (13), whereas other authors suggest that females have a better prognosis (3). These findings have not been demonstrated, neither in other studies $(3,6,14)$ nor in our study.

Large cohort studies regarding patients with CRC have demonstrated a surgical tumor resolution in $85 \%$ of patients(1). In our study, $40 \%$ of patients were classified as stages A or B -not considering "in situ" carcinomas as stage $\mathrm{A}-$ and $22 \%$ of them as stage $\mathrm{D}$, similar to findings by other authors $(8,13,15)$. General population studies establish the rate of Duke's stages A and B between 47 and $90 \%$ based on the consideration of in situ carcinoma as a stage-A tumor or -not respectively- the screening technique used. These rates are always higher than ours and other authors' in patients with symptoms $(13,16-20)$.

In summary, the only significant and determining variable for tumor extension is histologic differentiation. If diagnosis delay does not influence tumor extension at diagnosis, and the majority of wasted time is attributed to the patient delaying his or her seeing a doctor, the public health policy trying to accelerate the diagnostic process for CRC seems inefficient, and using screening techniques on the healthy population, which has demonstrated important decreases in mortality rate, is likely a better option (21).

The diagnosis of CRC in asymptomatic patients does not increase healthcare costs; instead, it reduces diagnosis- and treatment-related costs when compared to patients diagnosed during the symptomatic period (15).

\section{REFERENCES}

1. Mandel JS, Bond JH, Church TR, Snover DC, Bradley GM, Schuman LM, et al. Reducing mortality from colorectal cancer by screening for fecal occult blood. N Engl J Med 1993; 328: 1365-71.

2. Flashman K, O'Leary DP, Senapati A, Thompson MR. The Department of Health's "two week standard" for bowel cancer: is it working? Gut 2004; 53: 387-91.

3. Young CJ, Sweeney JL, Hunter A. Implications of delayed diagnosis in colorectal cancer. Aust N Z J Surg 2000; 70: 635-8.

4. Kiran PR, Glass RE. Duration of symptoms and spread of colorectal cancer: a short history does not mean early disease. Ann R Coll Surg Engl 2002; 84: 381-5.

5. Maté-Jiménez J, Sarbelio R, Pajares-García JM. Demora diagnóstica en el carcinoma de colon y recto: estudio prospectivo entre 1978 y 1982. Rev Esp Enferm Dig 1984; 66: 307-10.

6. Lebel E, Fraser D, Fraser GM, Niv Y. Colorectal cancer in the south of Israel; comparison of the clinical characteristics and survival between two periods, 1981-2 and 1986-7. Colorectal Dis 2003; 5: 13944.

7. McArdle CS, McMillan DC, Hole DJ. Male gender adversely affects survival following surgery for colorectal cancer. Br J Surg 2003; 90 : 711-5

8. Campbell NC, Elliott AM, Sharp L, Ritchie LD, Cassidy J, Little J. Rural and urban differences in stage at diagnosis of colorectal and lung cancers. Br J Cancer 2001; 84: 910-4.

9. Polednak AP. Inpatient hospital admission through an emergency department in relation to stage at diagnosis of colorectal cancer. Cancer Detect Prev 2000; 24: 283-9.

10. Koka VK, Potti A, Fraiman GN, Hanekom D, Hanley JF. An epidemiological study evaluating the relationship of distance from a tertiary care cancer center to early detection of colorectal carcinoma. Anticancer Res 2002; 22: 2481-3.

11. Takahashi K, Mori T, Yasuno M. Histologic grade of metastatic lymph node and prognosis of rectal cancer. Dis Colon Rectum 2000; 43: S40-6.

12. Massacesi C, Norman A, Price T, Hill M, Ross P, Cunningham D. A clinical nomogram for predicting long-term survival in advanced colorectal cancer. Eur J Cancer 2000; 36: 2044-52.

13. Chiang JM, Chen MC, Changchien CR, Chen JS, Tang R, Wang JY, et al. Favorable influence of age on tumor characteristics of sporadic colorectal adenocarcinoma: patients 30 years of age or younger may be a distinct patient group. Dis Colon Rectum 2003; 46: 904-10.

14. Makela J, Kiviniemi H, Laitinen S. Prognostic factors after surgery in patients younger than 50 years old with colorectal adenocarcinoma. Hepatogastroenterology 2002; 49: 971-5.

15. Ramsey SD, Mandelson MT, Berry K, Etzioni R, Harrison R. CancerAtributable cost of diagnosis and care for persons with screen-detected versus symptom-detected colorectal cancer. Gastroenterology 2003; 125: 1645-50.

16. Wong JM, Yen MF, Lai MS, Duffy SW, Smith RA, Chen TH. Progression rates of colorectal cancer by Dukes' stage in a high-risk group: analysis of selective colorectal cancer screening. Cancer J 2004; 10: 160-9.

17. UK Flexible Sigmoidoscopy Screening Trial Investigators. Single flexible sigmoidoscopy screening to prevent colorectal cancer: baseline findings of a UK multicentre randomised trial. Lancet 2002 13; 359: 1291-300

18. Hoffman A, Abcarian H. Six years of occult blood screening in an urban public hospital: concepts, methods, and reflections on approaches to reducing avoidable mortality among black Americans. J Natl Med Assoc 1991; 83: 994-9.

19. Morris JB, Stellato TA, Guy BB, Gordon NH, Berger NA. A critica analysis of the largest reported mass fecal occult blood screening program in the United States. Am J Surg 1991; 161: 101-5.

20. Winawer SJ, Bond JF, editors. Cancer of the colon, rectum and anus. New York: McGraw-Hill; 1995. p. 279-90.

21. Mandel JS, Church TR, Bond JH, Ederer F, Geisser MS, Mongin SJ, et al. The effect of fecal occult-blood screening on the incidence of colorectal cancer. N Engl J Med 2000 30; 343: 1603-7. 